\title{
Enforcing the Scientific Method
}

\author{
Allen L. Brown, Jr. \\ Health Solutions Group, Microsoft Corporation
}

Within Microsoft's Health Solutions Group we are engaged in the development of a platform to assist life sciences researchers - a class of extreme knowledge worker. We refer to this platform by the rubric, Pharos. Pharos has many objectives. One of the most important of those objectives is to supply an audit trail for research. This audit trail serves primarily to provide researchers and Pharos with a shared understanding of both the conduct of a scientific investigation and the results of a scientific investigation. But there are also other stakeholders in the audit trail, including regulatory agencies, funding agencies, tenure granting institutions and for-profit research managements. Put another way, Pharos is also concerned with the rigorous enforcement of the scientific method. In this presentation I will examine the roles and interplay of audited inference and audited workflow in constructing an audit trail. 